\title{
Embedded Based Low Cost Pulse Oximeter
}

\author{
S.Prince Samuel ${ }^{1}$, B.Thilagavthi ${ }^{2}$ \\ ${ }^{1}$ M.Tech (Embedded Systems), Dept of ElE, Karunya University, Coimbatore, India, \\ ${ }^{2}$ Assistant Professor, Dept. of EIE, Karunya University, Coimbatore, India,
}

\begin{abstract}
This paper deals with the Measurement of heart rate and oxygen saturation. The developed real-time pulse monitoring system has a pair of light-emitting diodes (LEDs), one LED has a wavelength in red and other is in infrared region. The light is detected by a photodiode. The LEDs and photodiode are packed in Velcro strip such that it faces the patient's fingertip. The pulse data is calculated by Atmegal6 microcontroller unit. Pulse data is read every second and stored in microcontroller. The Measured Parameters were displayed in the LCD Module.
\end{abstract}

Keywords: Heart rate, Microcontroller, Oxygen Saturation, Pulse Oximeter.

\section{Introduction}

The very important parameters for health monitoring are heart rate and oxygen saturation. A Pulse Oximeter is a medical device that indirectly monitors the oxygen saturation of a patient's blood (as opposed to measuring oxygen saturation directly through a blood sample) and changes in blood volume in the skin, producing a Photoplethysmograph. Use of light to measure blood oxygen saturation and heart rate is called Pulse Oximeter. It relies on measurement of physiological signal called Photoplethysmograph (PPG), which is an optical measurement of the change in blood volume in the arteries. Pulse Oximeter requires PPG signals by radiating two wavelengths of light through the tissues and compares the light absorption characteristics of blood at different wavelengths. The comparison leads to measurement of the oxygenation of blood and is reported as blood oxygen saturation.

\section{Pulse Oximeter}

The Pulse Oximeter consists of pair of LEDs, red and infrared of two different wavelengths $(660 \mathrm{~nm}$ and $940 \mathrm{~nm}$ )connected back to back and the other side it has a photo detector. By placing the finger in between the LED the ratio (R) between the intensities of two different lights is measured. A Pulse Oximeter is a medical device that indirectly monitors the oxygen saturation of a patient's blood (as opposed to measuring oxygen saturation directly through a blood sample) and changes in blood volume in the skin, producing a Photoplethysmograph.

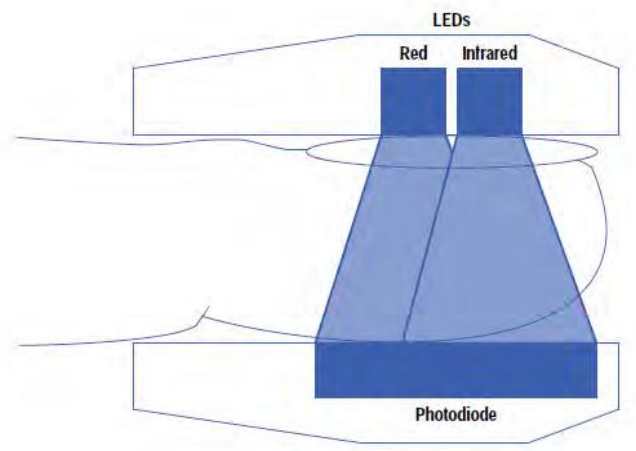

Fig. $1 \mathrm{SpO}_{2}$ Sensor

The overall block diagram consists of:

\section{Block Diagram Description}

a. Heartbeat Sensor

b. LED driver and signal conditioning circuit.

c. Atmega Microcontroller Development Board.

d. LCD Module. 


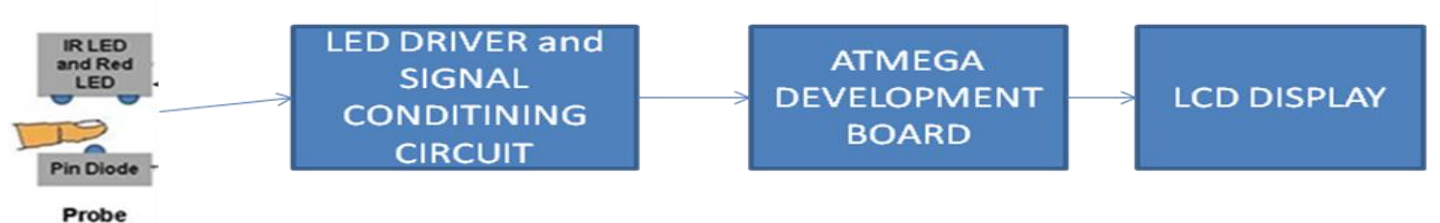

Fig. 2 Block Diagram of Heart rate Measurement

The developed real-time Embedded Based monitoring system has a pair of light-emitting diodes (LEDs), one LED has a wavelength in red and other is in infrared region. The light was detected by a photodiode. The LEDs and photodiode are packed in Velcro strip such that it faces the patient's fingertip. The signal from the sensor is amplified and filtered and then sent to the microcontroller for measuring the levels. The data from microcontroller unit is sent to LCD and then to PC via serial transmission. Oximeter probes are designed to have multiple light sources (or different colors of LEDs) that are switched on/off during the measurement by the instrument. From this method, the $\mathrm{SpO}_{2}$ and heart rate are calculated from detection of two kinds of light, i.e infrared and red LEDs.

\section{Transmission Vs Reflectance}

There are two approaches for developing an Oximetry probe. The first uses transmitted light, the second uses reflected light as shown in Fig.3.

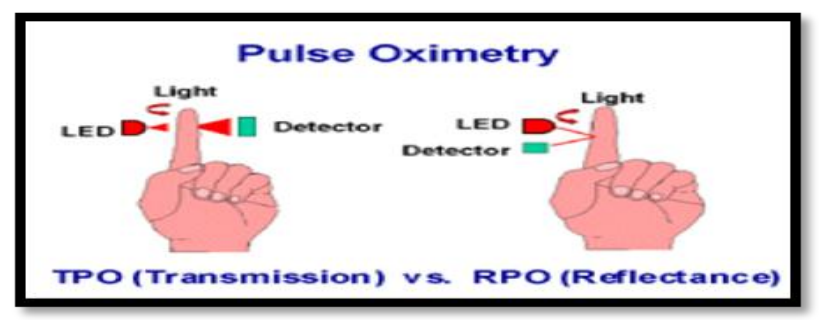

Fig.3 Transmission vs. Reflectance

A transmittance probe has two LEDs on one side and a photodiode (light detector) on the other. The tissue to be analyzed (commonly a finger or an ear) is inserted between the two. A reflectance probe has the LEDs and the photodiode(s) on the same side. It must be placed over a point with underlying bone. Light is emitted by the LEDs, passes through tissue and blood vessels, reflects from the bone, passes through the tissues again, and is then detected. Probes utilizing reflectance have the advantage that, regardless of the patient's size (infants to very large adults), the attachment site is always similar. However, attachment of a reflectance probe is, in general, more difficult than attachment of a transmittance probe, and proper attachment of a reflectance probe is essential to ensure its signal quality. Also, properly attaching a reflectance probe is quite time consuming which may lead to improper use. Both the transmittance and the reflectance probes are used clinically, though the transmittance probe is more common due to the convenience of attachment, and better signal quality.

\section{Calculation Of The Oxygen Level And Heart Beat Rate}

The transmitted of light through the tissue (in this research we use fingertip) can be calculated using Beer-Lambert Law (1) as follow:

$$
\text { lout }=\operatorname{Iin} \times e^{-A}
$$

Oximeter probes are designed to have multiple light sources (or different colors of LEDs) that are switched on/off during the measurement by the instrument. From this method, heart rate and oxygen saturation are detected from two kinds of light i.e infrared and red. The signal from two kinds of light has both AC and DC component. The AC and DC component from each of the wavelength need to be measured and the equation used for calculation is as follows:

$$
R=\frac{A C_{\text {red }} / D C_{\text {red }}}{A C_{i \bar{r}} / D C_{i r r}}
$$


$R$ is ratio between infrared and red light. The $\mathrm{SpO} 2$ can be calculated from calibrated equation(3) is as follows:

$$
\operatorname{Sp} 02=110-25 R \%
$$

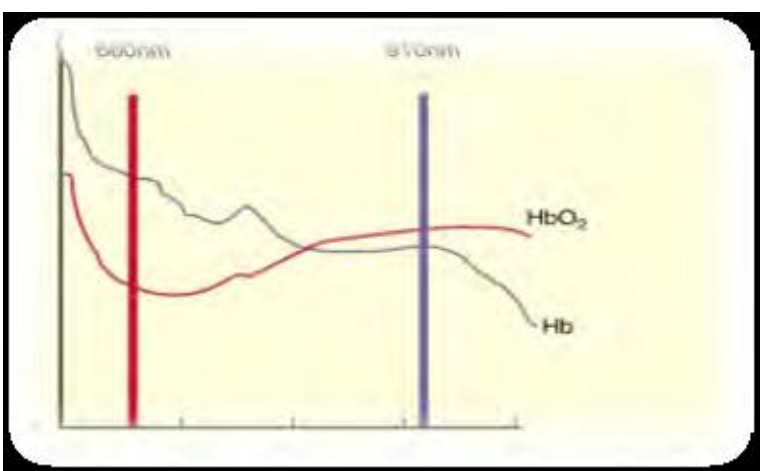

Fig 4. Absorption of Oxygenated and Non-oxygenated Haemoglobin at Different Wavelengths.

The graph above shows spectroscopic and absorptive properties (regarding which colour is absorbed) of oxygenated haemoglobin ( $\mathrm{HbO} 2)$ and deoxygenated haemoglobin $(\mathrm{Hb})$. Oxygenated haemoglobin absorbs more infrared light while deoxygenated blood absorbs more red light [1]. The heart beat is measured by counting the number of samples. A sensor is placed on a thin part of the patient's body, usually a fingertip or earlobe, or in the case of an infant, across a foot, and a light containing both red and infrared wavelengths is passed from one side to the other. Thus by using a single medical device we can measure both Oxygen saturation and Heart Rate of a human. The heart rate is determined by measuring the elapsed time (4) between peaks of the IR signal. The normal person $\mathrm{SpO}_{2}$ reading must be in the range of 95-100[2]. Thus by using a single medical device we can measure both Oxygen saturation and Heart Rate of a human The heart rate is then calculated using the equation as follows :

$$
B P M=60 / \text { periods (seconds) }
$$

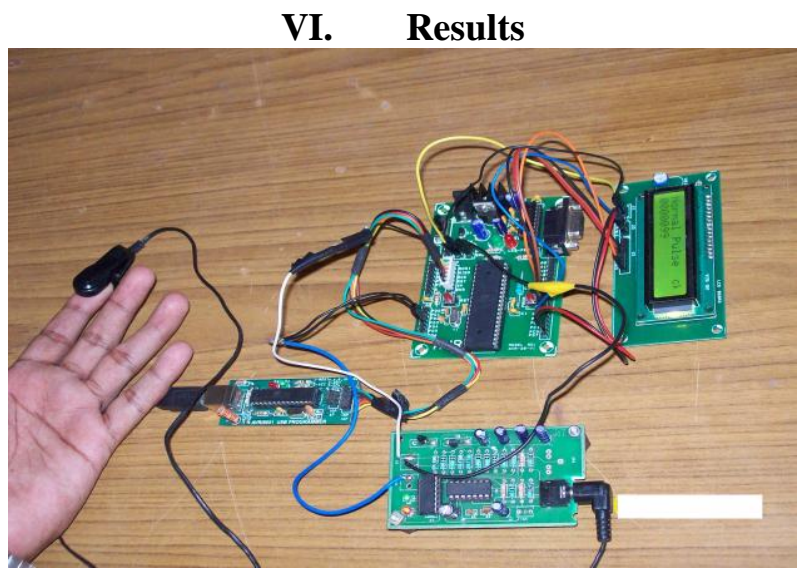

Fig.5 Sensing the signal and displaying the result in LCD

\section{Applications}

Pulse Oximeters were first used for vital sign monitoring during operations and anaesthesia. Since the device is non-invasive and allows immediate and real time monitoring, its use has expanded to include other purposes such as screening, diagnosis, patient follow-up, and self-monitoring. The Pulse Oximeter are used in determining the severity of a disease. The severity of a disease can be determined by clinical symptoms including $\mathrm{SpO}_{2}$. Blood gas analysis in Deciding whether to perform blood gas analysis in order to better understand the patient's condition .Pulse Oximeter is also used for home oxygen therapy, prescribing oxygen, and educating patients receiving home oxygen therapy. The amount of oxygen required depends on each 
patient's condition. The physician should determine the source of oxygen to be used, the appropriate oxygen flow, inhalation method, time of inhalation, and the amount of gas well as during exertion and sleep. Patients receiving HOT should have checkups at least monthly and education/management training by their physicians, including monitoring of blood oxygen saturation. In addition, patients receiving long-term HOT should have over night monitoring of $\mathrm{SpO}_{2}$ regularly to confirm whether the level of $\mathrm{SpO}_{2}$ decreases during sleep. The Pulse Oximeter may be unable to detect an adequate signal during abnormal hemodynamic conditions. The Pulse Oximeter is one of the most important advances in non invasive monitoring because it provides a means of continuously and quickly assessing arterial blood oxygenation. It is easy to use and interpret, requires little setup time, and poses no additional risks to the patient. Pulse Oximetry may soon be a standard of practice for routine monitoring in any clinical setting in which the patient is at risk of hypoxemia.

\section{Conclusion}

Thus a embedded based low cost pulse Oximeter has been designed to monitor the heart rate and oxygen saturation. The future scope of this device is that measured parameters can be transferred to doctor using different modes of wireless transmission devices like Bluetooth, Zigbee Module [4]. Even though Pulse Oximeter have got many advantages it has got some limitations like Pulse Oximeter assumes that arterial blood is the only pulsatile absorber and other sources of fluctuations could contribute to erroneous readings [5]. Translucency and blood flow at the spot where the measurement is taken can affect the accuracy of a reading. For example, if the area is largely opaque or the blood flow is low or irregular, accurate readings would be difficult. Understanding this is important to interpret a pulse Oximeter reading. Low atmospheric pressure in high altitude has little effect on a pulse Oximeter. Under various conditions, a Pulse Oximeter would give inaccurate readings.

\section{References}

[1] Soroor Behbahani, Mohammad Ali Pishbin, "New Oxygenation Method Based On Pulse Oximeter", American Journal of Biomedical Engineering 2012, Vol No. 2, Issue No.4,pp 185-188,Jan 2012.

[2] Sangeeta Bagha, Laxmi Shaw, "A Real Time Analysis Of PPG Signal For Measurement Of Spo2 And Pulse Rate”, International Journal of Computer Applications, Vol No. 36,Issue No.11,pp 45-50, December 2011 .

[3] Md.Mokarrom Hossain, A.S.M.Mohsin, Md.Nasimul Islam Maruf, Md. Asaduzzaman Shoeb and Ifat Al-Baqee, "An Advanced Architecture \& Instrumentation For Developing The System Of Monitoring A Oxygen Saturation Of A Patient", International Journal of Engineering and Technology, Vol No.2, Issue No.6,pp.598-602, December 2010.

[4] N. Watthanawisuth, T. Lomas, A. Wisitsoraat, A.Tuantranont,"Wireleless Wearable Pulse Oximeter For Health Monitoring Using Zigbee Wireless Sensor Network", IEEE Transactions On Electronics and Computer Technology, Vol No.2, Issue No.3, July 1, 2010 .

[5] Mohamed A. Zaltum a, b, M. Shukri Ahmad a, Ariffuddin Joret c and M.Mahadi Abdul Jamil, "Design And Development Of A Portable Pulse Oximetry System”, International Journal of Integrated Engineering, Vol No.2, Issue No.4,pp 37-43,December 2010. 